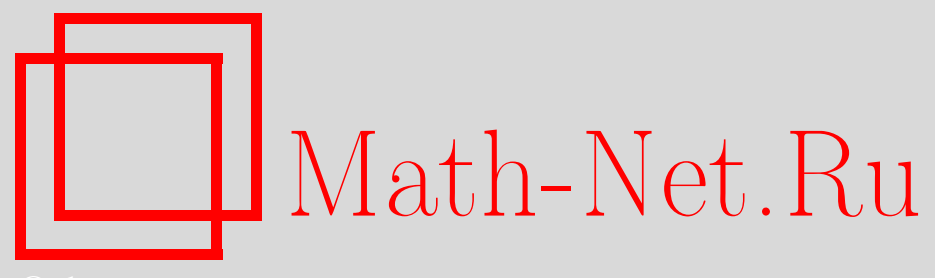

С. Крюков, Принцип суперпозиции в электростатике, Квант, 2019, номер 5, 41-44

DOI: https://doi.org/10.4213/kvant20190505

Использование Общероссийского математического портала Math-Net.Ru подразумевает, что вы прочитали и согласны с пользовательским соглашением http://www.mathnet.ru/rus/agreement

Параметры загрузки:

IP : 3.85 .73 .92

26 апреля 2023 г., 13:05:23

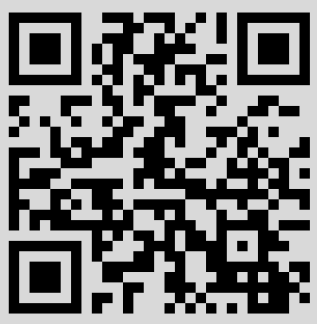




\section{Принцип \\ суперпозиции в электростатике}

\section{С.КРЮКОВ}

$\Pi$ РИНЦИП СУПЕРПОЗИЦИИ ЭЛЕКТРИЧЕСческих полей формулируется следующим образом:

Электрическое поле двух или нескольких точечных зарядов в любой точке пространства равно геометрической сумме полей, создаваемых в этой точке каждым зарядом в отдельности (т.е. когда остальные заряды устранены).

Главное содержание этого принципа состоит не в том, что поля складываются, как векторы, а в том, что они не искажаются присутствием других зарядов, причем не искажаются поля только точечных зарядов. Нетрудно привести пример, когда поля различных источников векторно суммируются, но принцип суперпозиции не выполняется. Если взять, скажем, два заряда, расположенных на проводниках, и найти поле в любой точке поблизости, то оно будет векторной суммой полей, созданных каждым зарядом, однако принцип суперпозиции здесь, конечно, не справедлив. Из-за неточечности источников за счет электризации через влияние распределения зарядов на поверхности проводников изменятся, и они будут создавать поля, отличные от тех, которые создавали бы, будучи уединенными. Таким образом, сложатся искаженные поля, т.е. требования принципа суперпозиции будут нарушены.

К сожалению, учащиеся далеко не всегда правильно понимают эту главную часть содержания принципа. Более того, порой и в учебной литературе при обсуждении (вполне корректном) процедуры сложения электрических полей упор делается именно на векторный характер этого сложения и при окончательной формулировке принципа су-

DOI: https://doi.org/10.4213/kvant20190505 перпозиции вопрос о независимости складываемых полей друг от друга просто оставляется в стороне (см., например, [1], c.51).

В этой статье на ряде примеров иллюстрируется применение принципа суперпозиции в электростатике. Естественно, любая электростатическая задача может быть решена общими методами, основанными на свойствах кулоновских полей и системе основных уравнений электростатики. Но порой эти решения оказываются весьма громоздкими. Однако если заданную в задаче систему зарядов удается представить в виде наложения более простых подсистем, не искажающих друг друга, то решение сложной задачи сводится к наложению решений более простых, иногда даже хорошо известных. Это позволяет не только быстро и красиво (порой устно) получить ответ, но и гораздо нагляднее представить структуру возбуждаемых данной системой зарядов полей. При этом чрезвычайно важно быть уверенным в том, что найденные подсистемы зарядов действительно не исказят друг друга и решения подзадач можно просто накладывать. В этом основная трудность использования данного метода, которая порой приводит к ошибкам.

Рассмотрим теперь некоторые конкретные задачи, поддающиеся решению указанным методом.

Задача 1. Центр проводящего шара радиусом а, несущего заряд $Q$, находится на расстоянии $R$ от точечного заряда q. Найдите потенциал $\varphi$ шара, если а и $R$ сравнимы друг с другом $(a<R)$.

Решение. Поскольку объем шара эквипотенциален, достаточно найти потенциал какой-либо его точки, например центра. Для этого разобьем заряд шара $Q$ на малые элементы (точечные заряды) и применим общую формулу для потенциала, созданного произвольным распределением заряда в любой точке поля $M$ :

$$
\varphi(M)=k \sum_{i=1}^{n} \frac{\Delta q_{i}}{r_{i}},
$$

где $k=\frac{1}{4 \pi \varepsilon_{0}}=9 \cdot 10^{9}$ ед. СИ, $\Delta q_{i}-i$-й точечный заряд, а $r_{i}$ - расстояние от него до исследуемой точки поля. В нашем случае 


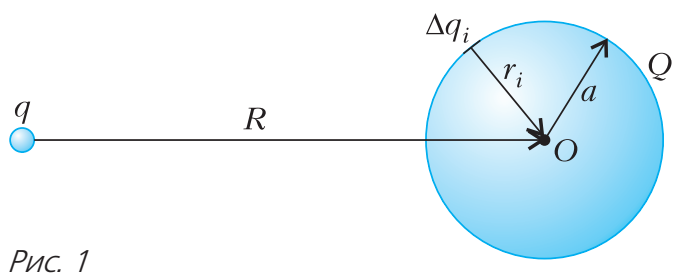

(рис.1) для зарядов $\Delta q_{i}$ на шаре все расстояния одинаковы: $r_{i}=a=$ const, так что

$$
\begin{aligned}
\varphi=k\left(\frac{q}{R}+\sum_{i=1}^{n} \frac{\Delta q_{i}}{a}\right)= \\
=k\left(\frac{q}{R}+\frac{1}{a} \sum_{i=1}^{n} \Delta q_{i}\right)=k\left(\frac{q}{R}+\frac{Q}{a}\right) .(*)
\end{aligned}
$$

Попытаемся теперь представить решение данной задачи в виде наложения решений двух более простых задач: незаряженный шар в поле точечного заряда и уединенный заряженный шар.

Вообще, единственным условием, которому подчиняется распределение зарядов на поверхности системы проводников, является равенство нулю напряженности поля в каждой точке внутри каждого проводника. Теорема единственности утверждает, что такое распределение всегда существует и оно единственно, т.е. других распределений, удовлетворяющих данному условию, нет.

Заряд $q$ индуцирует на незаряженном шаре некое неравномерное распределение зарядов с поверхностной плотностью $\sigma(M)$, причем, очевидно, система зарядов $\{q, \sigma(M)\}$ не создает поля внутри шара (рис.2). В этом

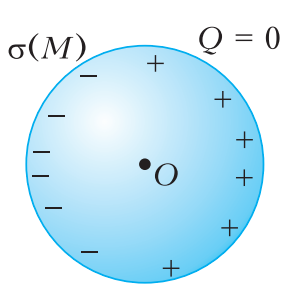

Pnc. 2 случае , полагая в (*) $\sum_{i=1}^{n} \Delta q_{i}=0$, получаем первое слагаемое ответа. (Точное решение - расчет поля $\vec{E}$ и $\sigma(M)-$ может быть получено методом изображений; см., например, [2], c.124-126.) Если теперь на уединенный шар поместить заряд $Q$, то он, распределившись равномерно по поверхности шара, тоже не даст поля внутри. Этой ситуации соответствует второе слагаемое ответа. Поскольку оба распределения (порознь) не дают поля внутри, простая их суперпозиция (без взаимного искажения) его там тоже не даст и, в силу теоремы единственности, никаких дру- гих распределений здесь возникнуть не может.

Замечание. Из проведенных рассуж$q$ дений следует, что при внесении незаря женной проводящей сферы (шара) в любое заданное поле на ней индуцируются заряды, поле которых накладывается на внешнее. В результате потенциалы всех точек поля изменятся, кроме одной. Это центр сферы, где потенциал сохранит прежнее значение.

Задача 2. Две проводящие сферы радиусами $r_{0}$ и $R=3 r_{0}$, расположенные одна внутpи другой, как показано на рисунке 3,

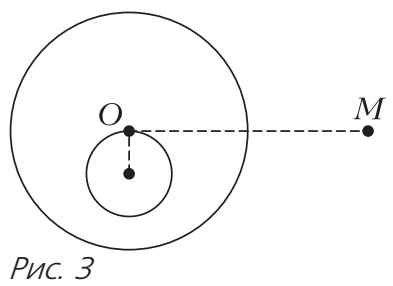

заряжены зарядами q и $Q$ соответственно. Найдите напряженность $\vec{E}$ и потенциал $\varphi$ электрического поля в точке $M$, отстоящей от чентра О большей сферы на расстояние $2 R$, которое отсчитано в направлении, перпендикулярном линии центров.

Решение. На первый взгляд задача кажется трудной: заряженные сферы будут влиять друг на друга, распределения зарядов на них окажутся неравномерными и непонятно, как искать поле и потенциал в точке $M$. Для прояснения ситуации рассмотрим более общую задачу: проводник произвольной формы заключен в замкнутую проводящую оболочку тоже произвольной формы, причем все это находится в окружении неких зарядов. Какие особенности будут иметь поля внутри и снаружи оболочки, если ее и проводник зарядить какими-то зарядами?

Поместим на проводник и окружающую его оболочку произвольные заряды $q_{1}$ и $q_{2}$ (рис.4,a). Эти заряды так разместятся на трех имеющихся поверхностях $S_{1}, S_{2}$ и $S_{3}$, что поле в каждой точке проводника и оболочки обратится в ноль. При этом заряд $q_{2}$ оболочки разделится вполне определенным образом между внутренней и внешней ее поверхностями: на внутренней появится заряд $-q_{1}$, а оставшийся заряд $+\left(q_{1}+q_{2}\right)$ уйдет на внешнюю. Действительно, выбирая замкнутую поверхность $S$, целиком находящую- 


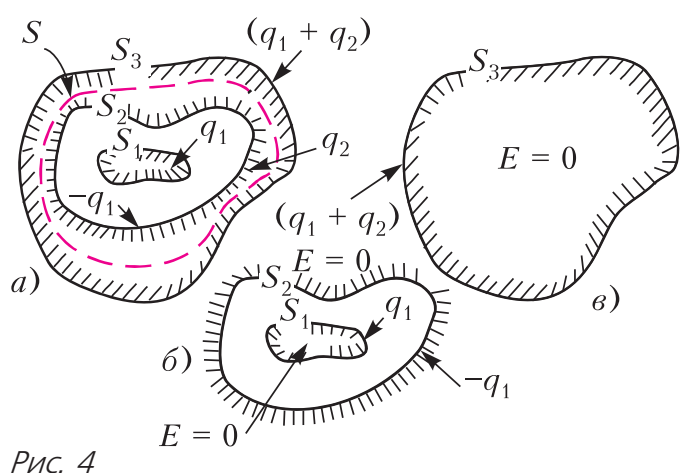

ся в толще оболочки (где $E=0)$, и применяя к ней теорему Гаусса, мы получим, что из равенства нулю потока через эту поверхность следует равенство нулю полного заряда внутри нее. Таким образом, если на проводнике находится заряд $q_{1}$, то заряд $-q_{1}$ должен появиться на внутренней поверхности оболочки.

Попытаемся теперь представить рассматриваемую картину распределения заряда (и созданного им поля) в виде суперпозиции картин двух более простых распределений, изображенных на рисунках 4,б и в. Первая картина получается, если все пространство снаружи оболочки заполнить проводником. При этом останутся лишь две поверхности $S_{1}$ и $S_{2}$, заряженные зарядами $q_{1}$ и $-q_{1}$, и поле между ними. Во всем остальном пространстве, т. е. внутри $S_{1}$ и снаружи $S_{2}$, система зарядов $\left\{q_{1},-q_{1}\right\}$ поля не создает. Для реализации второй картины заполним проводником всю область внутри оболочки. Тогда останется поверхность $S_{3}$ с зарядом $q_{1}+q_{2}$ и все остальные (внешние) заряды, размещенные вне оболочки произвольным образом. Эта система зарядов дает отличное от нуля поле только снаружи поверхности $S_{3}$, внутри нее в каждой точке $E=0$.

Итак, мы имеем две системы зарядов $\left\{q_{1},-q_{1}\right\}$ и $\left\{q_{1}+q_{2}\right.$, остальные заряды $\}$, причем первая система не создает поля там, где находятся заряды второй системы, а вторая не возбуждает его в местах расположения зарядов первой. Значит, при наложении этих распределений они не почувствуют друг друга, а потому никак друг друга не исказят. При этом поля их сложатся в каждой точке, и там, где оба распределения порознь давали нулевое поле, оно нулевым и останется. Следовательно, после наложения будут две области нулевого поля: внутри $S_{1}$ и между $S_{2}$ и $S_{3}$. Но это как раз области, занимаемые проводником и окружающей его оболочкой. Таким образом, найденное распределение заряда, давая нулевое поле внутри проводника и оболочки, является решением поставленной задачи и, в силу теоремы единственности, решением единственным.

Пространства внутри и вне оболочки оказываются совершенно не связанными друг с другом: любые перемещения проводника внутри оболочки, приводя лишь к перераспределению заряда на ее внутренней поверхности, никак не скажутся на полях и распределениях заряда снаружи и наоборот.

Вернемся теперь к нашей задаче. Из сказанного следует, что положение малой сферы внутри большой совершенно не влияет на поле снаружи. Оно будет создаваться равномерно распределенным по внешней поверхности большей сферы зарядом $q+Q$, т.е. будет сферически симметричным и определяться законом Кулона для точечного заряда указанной величины, расположенного в центре большой сферы. Стало быть, искомые величины напряженности и потенциала соответственно равны

$$
E=k \frac{q+Q}{4 R^{2}} \text { и } \varphi=k \frac{q+Q}{2 R} .
$$

Задача 3. K уединенному незаряженному плоскому воздушному конденсатору емкостью С снаружи подносят точечный заряд q, располагая его на расстоянии а, много меньшем размеров обкладок конденсатора, и далеко от их краев. Найдите напряжение U на конденсаторе.

Решение. При приближении точечного заряда $q$ к правой пластине конденсатора на ее наружной поверхности индуцируется заряд противоположного знака (см., например, [2], с.122-124), распределенный (неравномерно) с какой-то поверхностной плотностью $\sigma(M)$ (рис.5). Эта система зарядов $\{+q, \sigma(M)\}$ не создает поля нигде левее наружной поверхности правой обкладки (т.е. в толще пластин $E=0$ ). Используя метод отражений и теорему Гаусса, нетрудно показать, что полный наведенный заряд, соответствующий плотности $\sigma(M)$, в точности равен $-q$. Поскольку правая обкладка не заряжена, на ней высвобождается заряд $+q$, который уже будет создавать поле внутри (и 

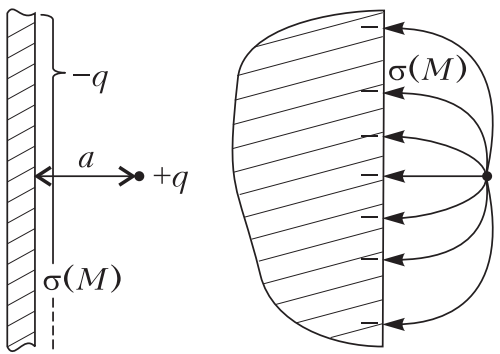

Pис. 5

вне) конденсатора и в свою очередь наведет на поверхностях левой обкладки некие заряды (в сумме равные нулю). Эти заряды вместе с высвободившимся зарядом $+q$ так распределятся на четырех имеющихся поверхностях, чтобы тоже не создавать поля в толще каждой из обкладок. Величины этих зарядов легко угадываются (хотя могут быть найдены с помощью элементарного расчета). Это, если идти слева направо, $+\frac{q}{2},-\frac{q}{2}$, $+\frac{q}{2}$ и $+\frac{q}{2}$ (рис.6). Так как ни одна из

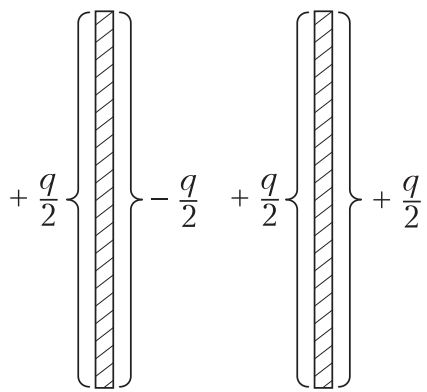

Pис. 6

приведенных подсистем зарядов не создает поля ни в одном из проводников, простая их (подсистем) суперпозиция (без взаимного искажения) его там тоже не даст и, стало быть, будет иметь место в действительности. Таким образом, поле внутри конденсатора возбуждается только зарядами $\pm \frac{q}{2}$ на внутренних сторонах обкладок, а значит,

$$
U=\frac{q / 2}{C}=\frac{q}{2 C} .
$$

Задача 4. $B$ точке $A$, расположенной на расстоянии $r$ от чентра $O$ незаряженной проводящей сферы радиусом $R$, находится точечный заряд q. Сферу заземляют длинным тонким проводником (рис.7). Насколь- ко изменится ( после заземления) потенциал ф точки В, являюшейся вершиной равностороннего треугольника $А B O$ ?

Решение. До заземления заряд $q$ индуцирует на сфере некое неравномерное распределение заряда с плот-

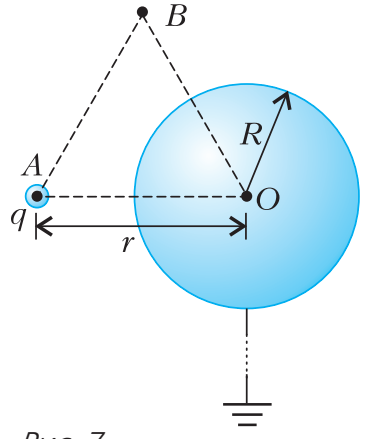

Pис. 7 ностью $\sigma(M)$, дающее вместе с $q$ нулевое поле в любой точке внутри проводника стенок сферы (а стало быть, и внутри всей сферы). После заземления на сфере появляется заряд $Q=-\frac{R}{r} q$ (зануляющий ее потенциал), который, очевидно, распределится равномерно по ее поверхности, поскольку только в этом случае он не создаст поля внутри сферы. Таким образом, появление заряда $Q$ не нарушит распределения напряженности и потенциала в пространстве, coзданные системой зарядов $\{q, \sigma(M)\}$, а лишь наложит на них собственные сферически симметричные распределения $\vec{E}(M)$ и $\varphi(M)$. Значит, потенциал точки $B$ изменится на величину

$$
\Delta \varphi=k \frac{Q}{r}=-k \frac{R q}{r^{2}} .
$$

Так же легко находим изменение вектора напряженности поля в точке $B$ :

$$
\Delta \vec{E}=k \frac{Q}{r^{2}} \vec{e}=-k \frac{R q}{r^{3}} \vec{e},
$$

где $\vec{e}-$ единичный вектор, направленный от $O$ к $B$.

Приведенные примеры, конечно, не исчерпывают все возможности применения принципа наложения при решении электростатических задач.

\section{Литература}

1. Г.Я.Мякишев, А.З.Синяков, Б.А.Слободсков. Физика. Электродинамика. 10-11 классы. Профильный уровень. - М.: Дрофа, 2007.

2. Р.Фейнман, Р.Лейтон, М.Сэндс. Фейнмановские лекции по физике. Выпуск 5. - М.: Мир, 1966. 